sciendo

\section{TATRA \\ MOUNTaiNS \\ Mathematical Publications}

DOI: $10.2478 /$ tmmp-2019-0020

Tatra Mt. Math. Publ. 74 (2019), 63-76

\title{
A THREE DIMENSIONAL MODIFICATION OF THE GAUSSIAN NUMBER FIELD
}

\author{
JÁN HALuŠKA - MAŁgorzata JASTRZĘBSKA \\ Mathematical Institute, Slovak Academy of Sciences, Košice SLOVAKIA \\ Institute of Mathematics and Physics, Siedlce University of Natural Sciences and Humanities, \\ Siedlce, POLAND
}

\begin{abstract}
For vectors in $\mathbf{E}_{3}$ we introduce an associative, commutative and distributive multiplication. We describe the related algebraic and geometrical properties, and hint some applications.

Based on properties of hyperbolic (Clifford) complex numbers, we prove that the resulting algebra $\mathbb{T}$ is an associative algebra over a field and contains a subring isomorphic to hyperbolic complex numbers. Moreover, the algebra $\mathbb{T}$ is isomorphic to direct product $\mathbb{C} \times \mathbb{R}$, and so it contains a subalgebra isomorphic to the Gaussian complex plane.
\end{abstract}

\section{Introduction - how to model colour vision?}

In a simplified explanation and for various purposes, three colours (red R, blue B, green G) satisfactorily model human colour vision.

The approximate utilization of complex plane structure is natural and commonly accepted because the eye retina is flat. In detail, a real vector space operations in the plane are sufficient for modelling the black-white vision. Operation of multiplication with linearly dependent R, G, B inputs could model the colour shade mixing. Such a planar approximation of vision is used in construction of colour TV-screens, colour photography, colour painting, etc. Note that all these kinds of illusions of reality in the human brain usually use two successive reflections.

(C) 2019 Mathematical Institute, Slovak Academy of Sciences. 2010 Mathematics Subject Classification: 12J05, 12D99, 11R52.

Keywords: Normed field, three dimensions, factor ring, generalized complex numbers. The paper is supported by VEGA Agency under grant 2/0106/19.

Licensed under the Creative Commons Attribution-NC-ND4.0 International Public License. 
In [3], a Gaussian complex plane spanned over three linearly dependent non collinear non-zero vectors is constructed. Having in mind the R, G, B colour decomposition of the white light, a point in the Gaussian complex plane is a sum of three colours of various intensity (the so-called wheel of colours in optics).

Via mathematics developed in this paper, we are able to work in the Euclidean three-dimensional geometrical space and the biological (human) vision is modelled as a unique reflection of light to a plane, biologically it means one projection to the retina. In [4], the R, G, B triples of colours are represented via functionals modifying the approach from [3].

In the present paper, an algebra $\mathbb{T}$ over field $\mathbb{R}$ is equipped with the basis $\left(\right.$ in $\left.\mathbb{E}_{3}\right)$

$$
\left\{\mathbf{1}_{\mathbb{T}}=(1,0,0), \mathbf{u}=(0,1,0), \mathbf{v}=(0,0,1)\right\}
$$

and the multiplication

\begin{tabular}{c|ccc}
$\otimes$ & $\mathbf{1}_{\mathbb{T}}$ & $\mathbf{u}$ & $\mathbf{v}$ \\
\hline $\mathbf{1}_{\mathbb{T}}$ & $\mathbf{1}_{\mathbb{T}}$ & $\mathbf{u}$ & $\mathbf{v}$ \\
$\mathbf{u}$ & $\mathbf{u}$ & $\mathbf{v}$ & $-\mathbf{1}_{\mathbb{T}}$ \\
$\mathbf{v}$ & $\mathbf{v}$ & $-\mathbf{1}_{\mathbb{T}}$ & $-\mathbf{u}$,
\end{tabular}

cf. Definition 1. It is isomorphic to the algebra $\mathbb{T}_{1}$ with the basis $\left\{k_{1}, k_{2}, k_{3}\right\}$, and the multiplication

\begin{tabular}{c|ccc}
$\cdot$ & $k_{1}$ & $k_{2}$ & $k_{3}$ \\
\hline$k_{1}$ & $k_{1}$ & $k_{2}$ & 0 \\
$k_{2}$ & $k_{2}$ & $-k_{1}$ & 0 \\
$k_{3}$ & 0 & 0 & $k_{3}$.
\end{tabular}

Thus, the algebra $\mathbb{T}$ is well-known and was studied for example in [1], 6], [2], [10].

Some of the results of the present paper are known.

The goal of this paper is mainly to show that

- the algebra $\mathbb{T}$ (with its arithmetic, geometrical and topological structures) is a spacial phenomenon (like $\mathbb{C}$ is a phenomenon in the plane);

- an annihilator $\mathbb{D}$ of the subalgebra $\mathbb{G}$ in algebra $\mathbb{T}$ (defined in the paper) is only a line in the space. Its all Lebesgue measurable subsets are of measure zero. Taking into account specific properties of the space $\mathbb{E}_{3}$, a specific (=spacial) infinitesimal analysis can be created for the Euclidean 3-dimensional space.

For the sake of the paper, let us remind some known facts.

Each associative division algebra over the real number field of finite dimension $n \in \mathbb{N}$ is isomorphic (1) to $\mathbb{R}$ (the field of all real numbers, $n=1$ ), or, (2) to $\mathbb{C}$ (the field of all Gaussian complex numbers, $n=2$ ), or, (3) to $\mathbb{H}$ (the algebra of all quaternions, $n=4$ ) by the 1877 theorem by G. F. Froben i us, cf. e.g., [7], p. 174. 


\section{A THREE DIMENSIONAL MODIFICATION OF THE GAUSSIAN NUMBER FIELD}

Let $\imath, \jmath, \varepsilon$ denote complex units for three types of complex numbers, respectively. Remind that for elliptic (Gaussian) numbers we have $\imath^{2}=-1,\|\imath\|=1$; for hyperbolic (Clifford) numbers we have $\jmath^{2}=1,\|\jmath\|=1$; and, for parabolic (Studdy) numbers we have $\varepsilon^{2}=0,\|\varepsilon\|=1$. Together all three types of complex numbers are called the generalized complex numbers. Under a simplified term complex numbers are usually understood the elliptic (Gaussian) complex numbers. For details and geometrical aspects of the generalized complex numbers, the reader is referred, e.g., to [5].

A Hausdorff topology on $\mathbb{T}$ is given via an absolute value, cf. Section 8 .

\section{Operation of multiplication of vectors}

Concerning operation of addition, it is known that elements of the space $\mathbb{E}_{3}$ form an additive group with null $(0,0,0) \stackrel{\text { def }}{=} \Lambda$. Let

$$
\mathbf{1}_{\mathbb{T}} \stackrel{\text { def }}{=}(1,0,0), \mathbf{u} \stackrel{\text { def }}{=}(0,1,0), \mathbf{v} \stackrel{\text { def }}{=}(0,0,1) .
$$

The set $\left\{\mathbf{1}_{\mathbb{T}}, \mathbf{u}, \mathbf{v}\right\}$ is a basis of the three dimensional vector space $\mathbb{E}_{3}$ over real line $\mathbb{R}$. So, every element $\mathbf{x} \in \mathbb{E}_{3}$ can be written as

$$
\mathbf{x} \stackrel{\text { def }}{=} X_{\mathbf{1}_{\mathbb{T}}} \mathbf{1}_{\mathbb{T}} \oplus X_{\mathbf{u}} \mathbf{u} \oplus X_{\mathbf{v}} \mathbf{v}
$$

where $X_{\mathbf{1}_{\mathbb{T}}}, X_{\mathbf{u}}, X_{\mathbf{v}} \in \mathbb{R}$. The sign $\oplus$ denotes an usual parallelepiped addition in the vector space $\mathbb{E}_{3}$ and the sign $\ominus$ denotes its inverse group operation; we write also $a \ominus b \stackrel{\text { def }}{=} a \oplus(\ominus b)$, so, $a \ominus a=\Lambda$.

DEFINITION 1. Let

Then,

$$
\mathbf{x}=\left(X_{\mathbf{1}_{\mathbb{T}}}, X_{\mathbf{u}}, X_{\mathbf{v}}\right) \in \mathbb{E}_{3} \quad \text { and } \quad \mathbf{y}=\left(Y_{\mathbf{1}_{\mathbb{T}}}, Y_{\mathbf{u}}, Y_{\mathbf{v}}\right) \in \mathbb{E}_{3}
$$

$$
\begin{aligned}
\mathbf{x} \otimes \mathbf{y} \stackrel{\text { def }}{=} \mathbf{1}_{\mathbb{T}}\left(X_{\mathbf{1}_{\mathbb{T}}} Y_{\mathbf{1}_{\mathbb{T}}}-\right. & \left.X_{\mathbf{u}} Y_{\mathbf{v}}-X_{\mathbf{v}} Y_{\mathbf{u}}\right) \\
& \oplus \mathbf{u}\left(X_{\mathbf{1}_{\mathbb{T}}} Y_{\mathbf{u}}+X_{\mathbf{u}} Y_{\mathbf{1}_{\mathbb{T}}}-X_{\mathbf{v}} Y_{\mathbf{v}}\right) \\
& \oplus \mathbf{v}\left(X_{\mathbf{1}_{\mathbb{T}}} Y_{\mathbf{v}}+X_{\mathbf{u}} Y_{\mathbf{u}}+X_{\mathbf{v}} Y_{\mathbf{1}_{\mathbb{T}}}\right) .
\end{aligned}
$$

Let $\mathbb{T}$ denote the vector space $\mathbb{E}_{3}$ over $\mathbb{R}$ equipped with this operation of multiplication.

Remark 1. The operation of multiplication in $\mathbb{E}_{3}$ can be equivalently introduced via the multiplication of basic elements as follows:

$$
\begin{aligned}
\mathbf{1}_{\mathbb{T}} \otimes \mathbf{1}_{\mathbb{T}}=\mathbf{1}_{\mathbb{T}}, \mathbf{u} \otimes \mathbf{u}=\mathbf{v}, \mathbf{v} \otimes \mathbf{v}=\ominus \mathbf{u} \\
\mathbf{1}_{\mathbb{T}} \otimes \mathbf{u}=\mathbf{u} \otimes \mathbf{1}_{\mathbb{T}}=\mathbf{u}, \mathbf{1}_{\mathbb{T}} \otimes \mathbf{v}=\mathbf{v} \otimes \mathbf{1}_{\mathbb{T}}=\mathbf{v}, \mathbf{u} \otimes \mathbf{v}=\mathbf{v} \otimes \mathbf{u}=\ominus \mathbf{1}_{\mathbb{T}} .
\end{aligned}
$$


For example, geometrically, the entries of the table are vertexes of the regular octahedron in $\mathbb{E}_{3}$, the structure of the operation of multiplication becomes clearly visible.

\begin{tabular}{c||ccc|ccc}
$\otimes$ & $\mathbf{1}_{\mathbb{T}}$ & $\mathbf{u}$ & $\mathbf{v}$ & $-\mathbf{1}_{\mathbb{T}}$ & $-\mathbf{u}$ & $-\mathbf{v}$ \\
\hline \hline $\mathbf{1}_{\mathbb{T}}$ & $\mathbf{1}_{\mathbb{T}}$ & $\mathbf{u}$ & $\mathbf{v}$ & $-\mathbf{1}_{\mathbb{T}}$ & $-\mathbf{u}$ & $-\mathbf{v}$ \\
$\mathbf{u}$ & $\mathbf{u}$ & $\mathbf{v}$ & $-\mathbf{1}_{\mathbb{T}}$ & $-\mathbf{u}$ & $-\mathbf{v}$ & $\mathbf{1}_{\mathbb{T}}$ \\
$\mathbf{v}$ & $\mathbf{v}$ & $-\mathbf{1}_{\mathbb{T}}$ & $-\mathbf{u}$ & $-\mathbf{v}$ & $\mathbf{1}_{\mathbb{T}}$ & $\mathbf{u}$ \\
\hline$-\mathbf{1}_{\mathbb{T}}$ & $-\mathbf{1}_{\mathbb{T}}$ & $-\mathbf{u}$ & $-\mathbf{v}$ & $\mathbf{1}_{\mathbb{T}}$ & $\mathbf{u}$ & $\mathbf{v}$ \\
$-\mathbf{u}$ & $-\mathbf{u}$ & $-\mathbf{v}$ & $\mathbf{1}_{\mathbb{T}}$ & $\mathbf{u}$ & $\mathbf{v}$ & $-\mathbf{1}_{\mathbb{T}}$ \\
$-\mathbf{v}$ & $-\mathbf{v}$ & $\mathbf{1}_{\mathbb{T}}$ & $\mathbf{u}$ & $\mathbf{v}$ & $-\mathbf{1}_{\mathbb{T}}$ & $-\mathbf{u}$.
\end{tabular}

Definition 2. Let $\mathbb{K}$ be a field. An algebra over $\mathbb{K}$ is a vector space $A$ over $\mathbb{K}$ together with a bilinear associative multiplication (denoted by ·).

In other words, for arbitrary elements $a, b, c$ from a vector space $A$ and for arbitrary $\lambda$ from $\mathbb{K}$, the following equalities are satisfied:

1) $a \cdot(b+c)=a \cdot b+a \cdot c$;

2) $(b+c) \cdot a=b \cdot a+c \cdot a$

3) $(a \cdot b) \cdot c=a \cdot(b \cdot c)$;

4) $(\lambda a) \cdot b=a \cdot(\lambda b)=\lambda(a \cdot b)$.

Algebras over a field which also satisfy commutativity for multiplication are called commutative algebras over a field. An algebra $A$ is said to be finite dimensional or infinite dimensional according to whether the space $A$ is finite dimensional or infinite dimensional. An algebra $A$ is unital if it has an identity (unit) element with respect to the multiplication. An ideal of unital algebra $A$ is a linear subspace which is also an ideal in $A$ as a ring.

It follows from the bilinearity of the multiplication in algebra over a field that, given a basis $\left\{a_{1}, a_{2}, \ldots, a_{n}\right\}$ of the space $A$, the multiplication is uniquely determined by the products of the basic vectors $a_{i} \cdot a_{j}$. It is sufficient to prove associativity of multiplication only for basic vectors. For more details on associative algebras over a field, we refer the reader to [2], 9].

Theorem 1. The algebra $\mathbb{T}$ is three-dimensional unital associative and commutative algebra over field $\mathbb{R}$.

P r o of. The commutativity of multiplication and the distributivity can be easily checked from the definition of algebra $\mathbb{T}$. It is easy to see that identity element in $\mathbb{T}$ is equal to $\mathbf{1}_{\mathbb{T}}=(1,0,0)$. Proof of the associativity

$$
[\mathbf{x} \otimes \mathbf{y}] \otimes \mathbf{z}=\mathbf{x} \otimes[\mathbf{y} \otimes \mathbf{z}]
$$

needs a rather longer but only technical calculations. 


\section{A THREE DIMENSIONAL MODIFICATION OF THE GAUSSIAN NUMBER FIELD}

\section{3. $\sigma$-Conjugation, a homomorphism of $\mathbb{T}$ to $\sigma$}

Definition 3. If

$$
\mathbf{x}=\mathbf{1}_{\mathbb{T}} X_{\mathbf{1}_{\mathbb{T}}} \oplus \mathbf{u} X_{\mathbf{u}} \oplus \mathbf{v} X_{\mathbf{v}} \in \mathbb{T},
$$

then we define a $\sigma$-conjugate element $\mathbf{x}^{*}$ of the element $\mathbf{x}$ as follows

$$
\mathbf{x}^{*} \stackrel{\text { def }}{=} \mathbf{1}_{\mathbb{T}} X_{\mathbf{1}_{\mathbb{T}}} \ominus \mathbf{u} X_{\mathbf{v}} \ominus \mathbf{v} X_{\mathbf{u}} \in \mathbb{T}
$$

where $X_{\mathbf{1}_{\mathbb{T}}}, X_{\mathbf{u}}, X_{\mathbf{v}} \in \mathbb{R}$

Note that the vectors $\delta \stackrel{\text { def }}{=} \mathbf{u} \ominus \mathbf{v}$ and $\mathbf{1}_{\mathbb{T}}$ are perpendicular: the scalar product

$$
<\mathbf{u} \ominus \mathbf{v}, \mathbf{1}_{\mathbb{T}}>=<(0,1,-1),(1,0,0)>=0 .
$$

LEMMA 1. If

$$
\mathbf{x}=\mathbf{1}_{\mathbb{T}} X_{\mathbf{1}_{\mathbb{T}}} \oplus \mathbf{u} X_{\mathbf{u}} \oplus \mathbf{v} X_{\mathbf{v}} \in \mathbb{T}
$$

then

where

$$
\mathbf{x} \otimes \mathbf{x}^{*}=A(\mathbf{x}) \mathbf{1}_{\mathbb{T}} \oplus B(\mathbf{x}) \delta,
$$

$$
\begin{array}{cl}
A(\mathbf{x})=X_{\mathbf{1}_{\mathbb{T}}}^{2}+X_{\mathbf{u}}^{2}+X_{\mathbf{v}}^{2}, & B(\mathbf{x})=X_{\mathbf{1}_{\mathbb{T}}} X_{\mathbf{u}}+X_{\mathbf{u}} X_{\mathbf{v}}-X_{\mathbf{v}} X_{\mathbf{1}_{\mathbb{T}}}, \\
\delta=\mathbf{u} \ominus \mathbf{v} \quad \text { and } \quad & X_{\mathbf{1}_{\mathbb{T}}}, X_{\mathbf{u}}, X_{\mathbf{v}} \in \mathbb{R} .
\end{array}
$$

Pr o o f. By Definition 1.

$$
\begin{aligned}
\mathbf{x} \otimes \mathbf{x}^{*} & =\left[\mathbf{1}_{\mathbb{T}} X_{\mathbf{1}_{\mathbb{T}}} \oplus \mathbf{u} X_{\mathbf{u}} \oplus \mathbf{v} X_{\mathbf{v}}\right] \otimes\left[\mathbf{1}_{\mathbb{T}} X_{\mathbf{1}_{\mathbb{T}}} \ominus \mathbf{u} X_{\mathbf{v}} \ominus \mathbf{v} X_{\mathbf{u}}\right] \\
& =\mathbf{1}_{\mathbb{T}}\left(X_{\mathbf{1}_{\mathbb{T}}}^{2}+X_{\mathbf{u}}^{2}+X_{\mathbf{v}}^{2}\right) \oplus \delta\left(X_{\mathbf{1}_{\mathbb{T}}} X_{\mathbf{u}}+X_{\mathbf{u}} X_{\mathbf{v}}-X_{\mathbf{v}} X_{\mathbf{1}_{\mathbb{T}}}\right) .
\end{aligned}
$$

Recall that a subalgebra of an algebra $A$ over field $\mathbb{K}$ is a subset of elements that is closed under addition, multiplication, and scalar multiplication.

Theorem 2. Let $\sigma$ denote the linear subspace of $\mathbb{E}_{3}$ spanned by vectors $\mathbf{1}_{\mathbb{T}}$ and $\delta$. Let $\otimes$ be the restriction of $\otimes$ on the subspace $\sigma$ and let

$$
\mathbf{j}_{\mathbb{T}}=\frac{\mathbf{1}_{\mathbb{T}}}{3} \oplus \frac{2 \delta}{3}=\frac{1}{3}\left[\mathbf{1}_{\mathbb{T}} \oplus 2 \mathbf{u} \ominus 2 \mathbf{v}\right] .
$$

Then, $\sigma$ is a subalgebra of algebra $\mathbb{T}$ and the operation $\otimes$ is a hyperbolic complex multiplication on a plane $\sigma$. The, ,real" unit is $\mathbf{1}_{\mathbb{T}}$ and the, imaginary" unit is $\mathbf{j}_{\mathbb{T}}$, respectively, i.e.,

$$
\mathbf{j}_{\mathbb{T}} \otimes \mathbf{j}_{\mathbb{T}}=\mathbf{1}_{\mathbb{T}} .
$$

P r o of. In order to prove that $\sigma$ is a subalgebra of $\mathbb{T}$, it suffices to show that for all $\mathbf{x}, \mathbf{y} \in \sigma$ their product $\mathbf{x} \otimes \mathbf{y}=\mathbf{x} \oplus \mathbf{y}$ belongs to $\sigma$. This follows easily from direct calculations. A question of the length of an unit ,,imaginary" element $\mathbf{j}_{\mathbb{T}}$ will be solved after introducing a notion of the absolute value $\|\cdot\|$ on $\mathbb{T}$ such that $\left\|\mathbf{j}_{\mathbb{T}}\right\|=1$, cf. Theorem 4 , (vii). 


\section{JÁN HALUŠKA, MAŁGORZATA JASTRZĘBSKA}

To prove that $\sigma$ is a hyperbolic complex plane, it is sufficient to show that

$$
\mathbf{j}_{\mathbb{T}} \otimes \mathbf{j}_{\mathbb{T}}=\mathbf{1}_{\mathbb{T}},
$$

and this follows from direct calculations.

The following lemma is useful.

Lemma 2. If $\mathbf{x}, \mathbf{y} \in \mathbb{T}$, then $(\mathbf{x} \otimes \mathbf{y})^{*}=\mathbf{x}^{*} \otimes \mathbf{y}^{*}$.

Proof. Let us denote

and

$$
\mathbf{x}=\left(X_{\mathbf{1}_{\mathbb{T}}}, X_{\mathbf{u}}, X_{\mathbf{v}}\right), \quad \mathbf{y}=\left(Y_{\mathbf{1}_{\mathbb{T}}}, Y_{\mathbf{u}}, Y_{\mathbf{v}}\right)
$$

$$
\mathbf{x}^{*}=\left(X_{\mathbf{1}_{\mathbb{T}}}^{\prime}, X_{\mathbf{u}}^{\prime}, X_{\mathbf{v}}^{\prime}\right)=\left(X_{\mathbf{1}_{\mathbb{T}}},-X_{\mathbf{v}},-X_{\mathbf{u}}\right)
$$

and

$$
\mathbf{y}^{*}=\left(Y_{\mathbf{1}_{\mathbb{T}}}^{\prime}, Y_{\mathbf{u}}^{\prime}, Y_{\mathbf{v}}^{\prime}\right)=\left(Y_{\mathbf{1}_{\mathbb{T}}},-Y_{\mathbf{v}},-Y_{\mathbf{u}}\right),
$$

respectively. We have:

$$
\begin{aligned}
\mathbf{x}^{*} \otimes \mathbf{y}^{*}= & \mathbf{1}_{\mathbb{T}}\left(X_{\mathbf{1}_{\mathbb{T}}}^{\prime} Y_{\mathbf{1}_{\mathbb{T}}}^{\prime}-X_{\mathbf{u}}^{\prime} Y_{\mathbf{v}}^{\prime}-X_{\mathbf{v}}^{\prime} Y_{\mathbf{u}}^{\prime}\right) \\
& \oplus \mathbf{u}\left(X_{\mathbf{1}_{\mathbb{T}}}^{\prime} Y_{\mathbf{u}}^{\prime}+X_{\mathbf{u}}^{\prime} Y_{\mathbf{1}_{\mathbb{T}}}^{\prime}-X_{\mathbf{v}}^{\prime} Y_{\mathbf{v}}^{\prime}\right) \\
& \oplus \mathbf{v}\left(X_{\mathbf{1}_{\mathbb{T}}}^{\prime} Y_{\mathbf{v}}^{\prime}+X_{\mathbf{u}}^{\prime} Y_{\mathbf{u}}^{\prime}+X_{\mathbf{v}}^{\prime} Y_{\mathbf{1}_{\mathbb{T}}}^{\prime}\right) \\
= & \mathbf{1}_{\mathbb{T}}\left(X_{\mathbf{1}_{\mathbb{T}}} Y_{\mathbf{1}_{\mathbb{T}}}-X_{\mathbf{v}} Y_{\mathbf{u}}-X_{\mathbf{u}} Y_{\mathbf{v}}\right) \\
& \oplus \mathbf{u}\left(-X_{\mathbf{1}_{\mathbb{T}}} Y_{\mathbf{v}}-X_{\mathbf{v}} Y_{\mathbf{1}_{\mathbb{T}}}-X_{\mathbf{u}} Y_{\mathbf{u}}\right) \\
& \oplus \mathbf{v}\left(-X_{\mathbf{1}_{\mathbb{T}}} Y_{\mathbf{u}}+X_{\mathbf{v}} Y_{\mathbf{v}}-X_{\mathbf{u}} Y_{\mathbf{1}_{\mathbb{T}}}\right) \\
= & (\mathbf{x} \otimes \mathbf{y})^{*} .
\end{aligned}
$$

\section{A shift of the plane $\sigma$}

Let us make the following regular linear transformation of the plane $\sigma$ :

$$
\left(\mathbf{1}_{\mathbb{T}}, \delta\right) \rightarrow\left(\mathbf{1}_{\mathbb{T}}, \mathbf{j}_{\mathbb{T}}\right),
$$

where

Then,

$$
\mathbf{j}_{\mathbb{T}}=\frac{\mathbf{1}_{\mathbb{T}}}{3} \oplus \frac{2 \delta}{3}, \delta=\frac{3 \mathbf{j}_{\mathbb{T}}}{2} \ominus \frac{\mathbf{1}_{\mathbb{T}}}{2} .
$$

$$
\mathbf{x} \otimes \mathbf{x}^{*}=\mathcal{A}(\mathbf{x}) \mathbf{1}_{\mathbb{T}} \oplus \mathcal{B}(\mathbf{x}) \mathbf{j}_{\mathbb{T}},
$$

where

and

$$
\mathcal{A}(\mathbf{x})=\left(X_{\mathbf{1}_{\mathbb{T}}}^{2}+X_{\mathbf{u}}^{2}+X_{\mathbf{v}}^{2}\right)-\frac{X_{\mathbf{1}_{\mathbb{T}}} X_{\mathbf{u}}+X_{\mathbf{u}} X_{\mathbf{v}}-X_{\mathbf{1}_{\mathbb{T}}} X_{\mathbf{v}}}{2}
$$

$$
\mathcal{B}(\mathbf{x})=\frac{3\left(X_{\mathbf{1}_{\mathbb{T}}} X_{\mathbf{u}}+X_{\mathbf{u}} X_{\mathbf{v}}-X_{\mathbf{1}_{\mathbb{T}}} X_{\mathbf{v}}\right)}{2} .
$$




\section{A THREE DIMENSIONAL MODIFICATION OF THE GAUSSIAN NUMBER FIELD}

Remark 2. If it does not lead to ambiguities, we usually omit arguments in functions $A(\mathbf{x}), B(\mathbf{x}), \mathcal{A}(\mathbf{x}), \mathcal{B}(\mathbf{x})$ and white simply $A, B, \mathcal{A}, \mathcal{B}$, respectively.

Since $\mathbf{1}_{\mathbb{T}} \otimes \mathbf{1}_{\mathbb{T}}=\mathbf{1}_{\mathbb{T}}$ and $\mathbf{j}_{\mathbb{T}} \otimes \mathbf{j}_{\mathbb{T}}=\mathbf{1}_{\mathbb{T}}$, by Theorem 2 ,

$$
\begin{aligned}
\left(\mathcal{A} \mathbf{1}_{\mathbb{T}} \ominus \mathcal{B} \mathbf{j}_{\mathbb{T}}\right) \otimes\left(\mathcal{A} \mathbf{1}_{\mathbb{T}} \oplus \mathcal{B} \mathbf{j}_{\mathbb{T}}\right) & =\left[\left(\mathcal{A}^{2} \mathbf{1}_{\mathbb{T}} \otimes \mathbf{1}_{\mathbb{T}}\right) \ominus\left(\mathcal{B}^{2} \mathbf{j}_{\mathbb{T}} \otimes \mathbf{j}_{\mathbb{T}}\right)\right] \\
& =\mathcal{A}^{2} \mathbf{1}_{\mathbb{T}} \ominus \mathcal{B}^{2} \mathbf{1}_{\mathbb{T}}=\left(\mathcal{A}^{2}-\mathcal{B}^{2}\right) \mathbf{1}_{\mathbb{T}} \\
& =(\mathcal{A}+\mathcal{B}) \cdot(\mathcal{A}-\mathcal{B}) \mathbf{1}_{\mathbb{T}}
\end{aligned}
$$

\section{Expression $\mathcal{A}+\mathcal{B}$}

Lemma 3. Let $\mathbf{x}=\left(X_{\mathbf{1}_{\mathbb{T}}}, X_{\mathbf{u}}, X_{\mathbf{v}}\right) \in \mathbb{T}$. Then,

$$
\mathcal{A}+\mathcal{B}=A+B=\frac{\left(X_{\mathbf{1}_{\mathbb{T}}}+X_{\mathbf{u}}\right)^{2}}{2}+\frac{\left(X_{\mathbf{u}}+X_{\mathbf{v}}\right)^{2}}{2}+\frac{\left(X_{\mathbf{1}_{\mathbb{T}}}-X_{\mathbf{v}}\right)^{2}}{2},
$$

where

$$
A=X_{\mathbf{1}_{\mathbb{T}}}^{2}+X_{\mathbf{u}}^{2}+X_{\mathbf{v}}^{2}, \quad B=X_{\mathbf{1}_{\mathbb{T}}} X_{\mathbf{u}}+X_{\mathbf{u}} X_{\mathbf{v}}-X_{\mathbf{1}_{\mathbb{T}}} X_{\mathbf{v}} .
$$

Proof. It is easy to verify that

$$
\begin{aligned}
\mathcal{A}+\mathcal{B} & =A+B \\
& =\left(X_{\mathbf{1}_{\mathbb{T}}}^{2}+X_{\mathbf{u}}^{2}+X_{\mathbf{v}}^{2}\right)+\left(X_{\mathbf{u}} X_{\mathbf{v}}+X_{\mathbf{u}} X_{\mathbf{1}_{\mathbb{T}}}-X_{\mathbf{1}_{\mathbb{T}}} X_{\mathbf{v}}\right) \\
& =\frac{\left(X_{\mathbf{1}_{\mathbb{T}}}+X_{\mathbf{u}}\right)^{2}}{2}+\frac{\left(X_{\mathbf{u}}+X_{\mathbf{v}}\right)^{2}}{2}+\frac{\left(X_{\mathbf{1}_{\mathbb{T}}}-X_{\mathbf{v}}\right)^{2}}{2} .
\end{aligned}
$$

Definition 4. Let $\Gamma=\left(X_{\mathbf{1}_{\mathbb{T}}}, X_{\mathbf{u}}, X_{\mathbf{v}}\right) \in \mathbb{T}$. Denote

$$
\mathbb{D} \stackrel{\text { def }}{=}\{\Gamma \in \mathbb{T} \mid A(\Gamma)+B(\Gamma)\}=0\},
$$

where

and

$$
A(\Gamma)=X_{\mathbf{1}_{\mathbb{T}}}^{2}+X_{\mathbf{u}}^{2}+X_{\mathbf{v}}^{2}
$$

$$
B(\Gamma)=X_{\mathbf{1}_{\mathbb{T}}} X_{\mathbf{u}}+X_{\mathbf{u}} X_{\mathbf{v}}-X_{\mathbf{v}} X_{\mathbf{1}_{\mathbb{T}}} .
$$

Lemma 4. $\mathbb{D}=\{\Gamma \in \mathbb{T} \mid \exists \gamma \in \mathbb{R}, \Gamma=(\gamma,-\gamma, \gamma)\}$.

Proof. If

$\Gamma=(\gamma,-\gamma, \gamma), \gamma \in \mathbb{R}$, then $A(\Gamma)=-B(\Gamma)=3 \gamma^{2}$. So, $A(\Gamma)+B(\Gamma)=0$. Now, let $\Gamma \in D$. Then $A(\Gamma)+B(\Gamma)=0$, and from Lemma 3 it follows that the system of equations

$$
\left\{\begin{array}{l}
X_{\mathbf{1}_{\mathbb{T}}}+X_{\mathbf{u}}=0, \\
X_{\mathbf{u}}+X_{\mathbf{v}}=0, \\
X_{\mathbf{1}_{\mathbb{T}}}-X_{\mathbf{v}}=0,
\end{array}\right.
$$

is satisfied. Hence, $X_{\mathbf{1}_{\mathbb{T}}}=\gamma, X_{\mathbf{u}}=-\gamma, X_{\mathbf{v}}=\gamma$, for some $\gamma \in \mathbb{R}$. 


\section{JÁN HALUŠKA, MAŁGORZATA JASTRZĘBSKA}

Remark 3. Vectors $(\gamma,-\gamma, \gamma), \gamma \in \mathbb{R}$, form a line, it is a diagonal of the fourths and sixths octants of the space $\mathbb{E}_{3}$.

Lemma 5. Let $\mathbf{x}=\left(X_{\mathbf{1}_{\mathbb{T}}}, X_{\mathbf{u}}, X_{\mathbf{v}}\right) \in \mathbb{T}$. Then,

$$
\mathbf{x} \otimes \mathbf{x}^{*}=(A, B,-B)=(A+B) \mathbf{1}_{\mathbb{T}} \oplus \Gamma,
$$

where

$$
A=X_{\mathbf{1}_{\mathbb{T}}}^{2}+X_{\mathbf{u}}^{2}+X_{\mathbf{v}}^{2}, \quad B=X_{\mathbf{1}_{\mathbb{T}}} X_{\mathbf{u}}+X_{\mathbf{v}} X_{\mathbf{u}}-X_{\mathbf{1}_{\mathbb{T}}} X_{\mathbf{v}}
$$

and

$$
\Gamma=(-B, B,-B) \in \mathbb{D} .
$$

Pr o of. A verification is trivial.

\section{Expression $\mathcal{A}-\mathcal{B}$}

Lemma 6. Let $\mathbf{x}=\left(X_{\mathbf{1}_{\mathbb{T}}}, X_{\mathbf{u}}, X_{\mathbf{v}}\right) \in \mathbb{T}$. Then,

$$
A-B=\mathcal{A}-\mathcal{B}=\frac{\left(X_{\mathbf{1}_{\mathbb{T}}}-X_{\mathbf{u}}\right)^{2}}{2}+\frac{\left(X_{\mathbf{u}}-X_{\mathbf{v}}\right)^{2}}{2}+\frac{\left(X_{\mathbf{1}_{\mathbb{T}}}+X_{\mathbf{v}}\right)^{2}}{2} .
$$

Proof. We have

$$
\begin{aligned}
\mathcal{A}-\mathcal{B}=A-B & =\left(X_{\mathbf{1}_{\mathbb{T}}}^{2}+X_{\mathbf{u}}^{2}+X_{\mathbf{v}}^{2}\right)+\left(X_{\mathbf{1}_{\mathbb{T}}} X_{\mathbf{v}}-X_{\mathbf{u}} X_{\mathbf{v}}-X_{\mathbf{u}} X_{\mathbf{1}_{\mathbb{T}}}\right) \\
& =\frac{\left(X_{\mathbf{1}_{\mathbb{T}}}-X_{\mathbf{u}}\right)^{2}}{2}+\frac{\left(X_{\mathbf{u}}-X_{\mathbf{v}}\right)^{2}}{2}+\frac{\left(X_{\mathbf{1}_{\mathbb{T}}}+X_{\mathbf{v}}\right)^{2}}{2} .
\end{aligned}
$$

\section{Algebraic structure of $\mathbb{T}$}

Lemma 7. The set $\mathbb{D}$ is an ideal in the algebra $\mathbb{T}$.

P r o o f. From Lemma $4, \mathbb{D}$ is the one-dimensional linear space spanned by vector $(1,-1,1)$. Let $\Gamma=\gamma(1,-1,1) \in \mathbb{D}$, where $\gamma \in \mathbb{R}$. Let $\mathbf{x}=\left(X_{\mathbf{1}_{\mathbb{T}}}, X_{\mathbf{u}}, X_{\mathbf{v}}\right) \in \mathbb{T}$, where $X_{\mathbf{1}_{\mathbb{T}}}, X_{\mathbf{u}}, X_{\mathbf{v}} \in \mathbb{R}$. Then, $\Gamma \otimes \mathbf{x} \in \mathbb{D}$. Indeed,

$$
\begin{aligned}
\Gamma \otimes \mathbf{x} & =(\gamma,-\gamma, \gamma) \otimes\left(X_{\mathbf{1}_{\mathbb{T}}}, X_{\mathbf{u}}, X_{\mathbf{v}}\right) \\
& =\left(X_{\mathbf{1}_{\mathbb{T}}} \gamma-X_{\mathbf{u}} \gamma+X_{\mathbf{v}} \gamma,-X_{\mathbf{1}_{\mathbb{T}}} \gamma+X_{\mathbf{u}} \gamma-X_{\mathbf{v}} \gamma, X_{\mathbf{1}_{\mathbb{T}}} \gamma-X_{\mathbf{u}} \gamma+X_{\mathbf{v}} \gamma\right) \\
& =(\beta,-\beta, \beta) \in \mathbb{D},
\end{aligned}
$$

where $\beta=X_{\mathbf{1}_{\mathbb{T}}} \gamma-X_{\mathbf{u}} \gamma+X_{\mathbf{v}} \gamma \in \mathbb{R}$. Consequently, $\mathbb{D}$ is an ideal in $\mathbb{T}$. 


\section{A THREE DIMENSIONAL MODIFICATION OF THE GAUSSIAN NUMBER FIELD}

Lemma 8. The set $\mathbb{G}=(\alpha-\beta, \alpha, \beta) \in \mathbb{T} \mid \alpha \in \mathbb{R}, \beta \in \mathbb{R}\}$ is an ideal in $\mathbb{T}$.

P r o o f. It is easily seen that the set $\mathbb{G}$ is two-dimensional linear space spanned by vectors $(1,1,0)$ and $(-1,0,1)$. Let $\theta=(\alpha-\beta, \alpha, \beta) \in \mathbb{G}$, where $\alpha, \gamma \in \mathbb{R}$. Let $\mathbf{x}=\left(X_{\mathbf{1}_{\mathbb{T}}}, X_{\mathbf{u}}, X_{\mathbf{v}}\right) \in \mathbb{T}$. Then, $\theta \otimes \mathbf{x} \in \mathbb{G}$.

Indeed,

$$
\begin{aligned}
& \theta \otimes \mathbf{x}=(\alpha-\beta, \alpha, \beta) \otimes\left(X_{\mathbf{1}_{\mathbb{T}}}, X_{\mathbf{u}}, X_{\mathbf{v}}\right) \\
&=\left((\alpha-\beta) X_{\mathbf{1}_{\mathbb{T}}}-\alpha X_{\mathbf{v}}-\beta X_{\mathbf{u}},\right. \\
& \quad(\alpha-\beta) X_{\mathbf{u}}+\alpha X_{\mathbf{1}_{\mathbb{T}}}-\beta X_{\mathbf{v}}, \\
&\left.\quad(\alpha-\beta) X_{\mathbf{v}}+\alpha X_{\mathbf{u}}+\beta X_{\mathbf{v}}\right) \in \mathbb{G} .
\end{aligned}
$$

Consequently, $\mathbb{G}$ is an ideal in $\mathbb{T}$.

Theorem 3. Let $\mathbb{T}$ be an algebra described in Definition 1 , Then, $\mathbb{T}$ is the direct sum of ideals $\mathbb{D}$ and $\mathbb{G}$. Moreover, algebra $\mathbb{T}$ is isomorphic to $\mathbb{C} \times \mathbb{R}$.

P r o o f. From definitions of ideals $\mathbb{D}$ and $\mathbb{G}$ it follows that $\mathbb{D} \cap \mathbb{G}=\Lambda$. The ideal $\mathbb{D}$ is a division ring, so, by Frobenius theorem, it is isomorphic to $\mathbb{R}$. The identity element $\mathbf{1}_{\mathbb{D}}$ of $\mathbb{D}$ equals $\mathbf{1}_{\mathbb{D}}=\left(\frac{1}{3},-\frac{1}{3}, \frac{1}{3}\right)$. It is easy to verify that the identity element $\mathbf{1}_{\mathbb{G}}$ of $\mathbb{G}$ is equal to $\mathbf{1}_{\mathbb{G}}=\left(\frac{2}{3}, \frac{1}{3},-\frac{1}{3}\right)$. Moreover, it is trivial to calculate that the ideal $\mathbb{G}$ has an element

$$
\mathbf{i}_{\mathbb{G}}=\left(0, \sqrt{\frac{1}{3}}, \sqrt{\frac{1}{3}}\right)
$$

which satisfies $\mathbf{i}_{\mathbb{G}} \otimes \mathbf{i}_{\mathbb{G}}=-\mathbf{1}_{\mathbb{G}}$. This forces $\mathbb{G}$ to be isomorphic to complex numbers $\mathbb{C}$.

Let $\mathbb{A}$ be an algebra over a field $\mathbb{K}$ and $S \subset \mathbb{A}$. Recall that an annihilator of $S$ in $\mathbb{A}$, denoted $\operatorname{Ann}_{\mathbb{A}}(S)$, is the set of all elements $a \in \mathbb{A}$ such that $a \cdot s=0$ for all $s \in S$.

In the following, we list several simple facts resulting directly from the Theorem 3

\section{Corrolary 1.}

- $A n n_{\mathbb{T}}(\mathbb{D})=\mathbb{G}$ and $A n n_{\mathbb{T}}(\mathbb{G})=\mathbb{D}$.

- $\mathbf{y} \in \mathbb{T}$ is a zero divisor in $\mathbb{T}$ if and only if $\mathbf{y} \in \mathbb{D}$ or $\mathbf{y} \in \mathbb{G}$.

- The element $\mathbf{x} \in \mathbb{T}$ is invertible in the algebra $\mathbb{T}$ if and only if there exist $a, b, c \in \mathbb{R}$ such that

$$
\begin{aligned}
\mathbf{x}=a(1,1,0) \oplus b(-1,0,1) & \oplus c(1,-1,1), \\
& \text { where } a^{2}+b^{2}>0 \text { and } c^{2}>0 .
\end{aligned}
$$




\section{Absolute value on $\mathbb{T}$}

A Hausdorff topology on $\mathbb{T}$ is determined via an absolute value, cf. [8].

Definition 5. Let $\mathbf{x}=\left(X_{\mathbf{1}_{\mathbb{T}}}, X_{\mathbf{u}}, X_{\mathbf{v}}\right) \in \mathbb{T}$. An absolute value of the element $\mathbf{x}$ is a non-negative real number $\|\mathbf{x}\|: \mathbf{x} \rightarrow[0,+\infty)$ such that

where

$$
\|\mathbf{x}\|=\sqrt{A+B}
$$

$$
A=X_{\mathbf{1}_{\mathbb{T}}}^{2}+X_{\mathbf{u}}^{2}+X_{\mathbf{v}}^{2}, \quad B=X_{\mathbf{1}_{\mathbb{T}}} X_{\mathbf{u}}+X_{\mathbf{u}} X_{\mathbf{v}}-X_{\mathbf{v}} X_{\mathbf{1}_{\mathbb{T}}} .
$$

The absolute value has the following properties:

Theorem 4. Let $\mathbf{x}=\left(X_{\mathbf{1}_{\mathbb{T}}}, X_{\mathbf{u}}, X_{\mathbf{v}}\right), \mathbf{y}=\left(Y_{\mathbf{1}_{\mathbb{T}}}, Y_{\mathbf{u}}, Y_{\mathbf{v}}\right) \in \mathbb{T} ; \gamma \in \mathbb{R}, \Gamma \in \mathbb{D}$.

Then

(i) $\|\Gamma\|=0$;

(ii) $\|\gamma \mathbf{x}\|=|\gamma| \cdot\|\mathbf{x}\|$;

(iii) $\|\mathbf{x}\| \geq 0$;

(iv) $\|\mathbf{x} \otimes \mathbf{y}\|=\|\mathbf{x}\| \cdot\|\mathbf{y}\|$;

(v) $\|\Gamma \oplus \mathbf{x}\|=\|\mathbf{x}\|$;

(vi) $\left\|\mathbf{1}_{\mathbb{T}}\right\|=\|\mathbf{u}\|=\|\mathbf{v}\|=1$;

(vii) $\left\|\mathbf{j}_{\mathbb{T}}\right\|=1$;

(viii) $\|(0,-\gamma, \gamma)\|=\|(\gamma,-\gamma, 0)\|=\|(\gamma, 0, \gamma)\|=\|(\gamma, 0,0)\|=\|(0,-\gamma, 0)\|=$ $\|(0,0, \gamma)\|=|\gamma| \geq 0$

(ix) $\|\mathbf{x}\|=\sqrt{\left\|\mathbf{x} \otimes \mathbf{x}^{*}\right\|}$;

(x) $\|\mathbf{x}+\mathbf{y}\| \leq\|\mathbf{x}\|+\|\mathbf{y}\|$,

(xi) $\left\|\left(\frac{2}{3}, \frac{1}{3},-\frac{1}{3}\right)\right\|=1,\left\|\left(0, \sqrt{\frac{1}{3}} \sqrt{\frac{1}{3}}\right)\right\|=1$.

Proof.

(i) There exists $\gamma \in \mathbb{R}$ such that $\Gamma=(\gamma,-\gamma, \gamma)$ is a zero divisor. Then, $\|\Gamma\|=0$ from Definition 5 .

(ii) This statement trivially follows from Definition [5] and (3).

(iii) It is a simple consequence of Lemma 3

(iv) By Lemma 2

$$
(\mathbf{x} \otimes \mathbf{y}) \otimes(\mathbf{x} \otimes \mathbf{y})^{*}=(\mathbf{x} \otimes \mathbf{y}) \otimes\left(\mathbf{x}^{*} \otimes \mathbf{y}^{*}\right) .
$$

Applying Lemma 5 together with properties of multiplication and addition in $\mathbb{T}$, we may prove the following points: 


\section{A THREE DIMENSIONAL MODIFICATION OF THE GAUSSIAN NUMBER FIELD}

a) The right side of Formula (41) can be written as

$$
\begin{aligned}
& (\mathbf{x} \otimes \mathbf{y}) \otimes(\mathbf{x} \otimes \mathbf{y})^{*}= \\
& (A(\mathbf{x} \otimes \mathbf{y})+B(\mathbf{x} \otimes \mathbf{y})) \mathbf{1}_{\mathbb{T}} \oplus(-B(\mathbf{x} \otimes \mathbf{y}), B(\mathbf{x} \otimes \mathbf{y}),-B(\mathbf{x} \otimes \mathbf{y}))= \\
& (A(\mathbf{x} \otimes \mathbf{y})+B(\mathbf{x} \otimes \mathbf{y})) \mathbf{1}_{\mathbb{T}} \oplus \Gamma_{2},
\end{aligned}
$$

where $\Gamma_{2}=(-B(\mathbf{x} \otimes \mathbf{y}), B(\mathbf{x} \otimes \mathbf{y}),-B(\mathbf{x} \otimes \mathbf{y})) \in \mathbb{D}$.

b) The left side of Formula (4) can be written as

$$
\begin{aligned}
&(\mathbf{x} \otimes \mathbf{y}) \otimes\left(\mathbf{x}^{*} \otimes \mathbf{y}^{*}\right)=\left(\mathbf{x} \otimes \mathbf{x}^{*}\right) \otimes\left(\mathbf{y} \otimes \mathbf{y}^{*}\right)= \\
&\left((A(\mathbf{x})+B(\mathbf{x})) \mathbf{1}_{\mathbb{T}} \oplus(-B(\mathbf{x}), B(\mathbf{x}),-B(\mathbf{x}))\right) \otimes \\
&\left((A(\mathbf{y})+B(\mathbf{y})) \mathbf{1}_{\mathbb{T}} \oplus(-B(\mathbf{y}), B(\mathbf{y}),-B(\mathbf{x}))\right) .
\end{aligned}
$$

Now, by Lemma 7 this is equivalent to $(A(\mathbf{x})+B(\mathbf{x}))(A(\mathbf{y})+B(\mathbf{y})) \mathbf{1}_{\mathbb{T}}+\Gamma_{1}$ for some $\Gamma_{1} \in \mathbb{D}$.

c) In fact, vectors contained in $\mathbb{D}$ are linearly independent from vectors $\alpha \mathbf{1}_{\mathbb{T}}$ for any $\alpha \in \mathbb{R}$.

Now, comparing the final formulas in items a) and b), we get that

$$
A(\mathbf{x} \otimes \mathbf{y})+B(\mathbf{x} \otimes \mathbf{y})=(A(\mathbf{x})+B(\mathbf{x}))(A(\mathbf{y})+B(\mathbf{y}))
$$

Hence, $\|\mathbf{x} \otimes \mathbf{y}\|=\|\mathbf{x}\| \cdot\|\mathbf{y}\|$.

(v) Let $\Gamma=(\gamma,-\gamma, \gamma)$.

Then, $\|\mathbf{x} \oplus \Gamma\|=\|\mathbf{x}\|$ is a simple consequence of Lemma 3 .

(vi) $\left\|\mathbf{1}_{\mathbb{T}}\right\|=\|\mathbf{u}\|=\|\mathbf{v}\|=1$ can be calculated directly from definition;

(vii) $\mathbf{j}_{\mathbb{T}}=\frac{1}{3}\left[\mathbf{1}_{\mathbb{T}} \oplus 2 \mathbf{u} \oplus 2(-\mathbf{v})\right]=\frac{1}{3}[(1,0,0) \oplus 2(0,1,0) \oplus 2(0,0,-1)]$

$$
=\left(\frac{1}{3}, \frac{2}{3},-\frac{2}{3}\right) \text {, }
$$

and

$$
\begin{aligned}
\left\|\mathbf{j}_{\mathbb{T}}\right\| & =\sqrt{A+B} \\
& =\sqrt{\left[\left(\frac{1}{3}\right)^{2}+\left(\frac{2}{3}\right)^{2}+\left(-\frac{2}{3}\right)^{2}\right]+\left[\frac{1}{3} \cdot \frac{2}{3}-\frac{2}{3} \cdot \frac{2}{3}+\frac{2}{3} \cdot \frac{1}{3}\right]}=1
\end{aligned}
$$

(viii) By item (v) and Definition 5 ,

(ix) By item (v) and Lemma 5

$$
\begin{aligned}
\sqrt{\left\|\mathbf{x} \otimes \mathbf{x}^{*}\right\|} & =\sqrt{\left\|(A+B) \mathbf{1}_{\mathbb{T}} \oplus(-B, B,-B)\right\|} \\
& =\sqrt{\left\|(A+B) \mathbf{1}_{\mathbb{T}}\right\|}=\sqrt{A+B}=\|\mathbf{x}\| ;
\end{aligned}
$$

(x) If $\mathbf{x}=\mathbf{y}=\Lambda$, then the triangle inequality holds trivially. 


\section{JÁN HALUŠKA, MAŁGORZATA JASTRZĘBSKA}

If one of coordinates of $\mathbf{x}$ and $\mathbf{y}$ is non-zero, then apply the item (v) such that without loss of generality we may suppose that both $\mathbf{x}, \mathbf{y}$ are of the following form

$$
\mathbf{x}=\left(0, X_{\mathbf{u}}, X_{\mathbf{v}}\right), \quad \mathbf{y}=\left(0, Y_{\mathbf{u}}, Y_{\mathbf{v}}\right), \quad \text { respectively. }
$$

Hence, also

$$
\mathbf{x}+\mathbf{y}=\left(0, X_{\mathbf{u}}+Y_{\mathbf{u}}, X_{\mathbf{v}}+Y_{\mathbf{v}}\right)
$$

By Definition 5 and Lemma 3 .

$$
\begin{aligned}
\|\mathbf{x}\| & =\sqrt{X_{\mathbf{u}}^{2}+X_{\mathbf{v}}^{2}+X_{\mathbf{u}} X_{\mathbf{v}}} \\
& =\sqrt{\frac{X_{\mathbf{u}}^{2}}{2}+\frac{X_{\mathbf{v}}^{2}}{2}+\frac{\left(X_{\mathbf{u}}+X_{\mathbf{v}}\right)^{2}}{2}} .
\end{aligned}
$$

Analogously,

$$
\|\mathbf{x}\|=\sqrt{\frac{Y_{\mathbf{u}}^{2}}{2}+{\frac{Y_{\mathbf{v}}}{2}}^{2}+\frac{\left(Y_{\mathbf{u}}+Y_{\mathbf{v}}\right)^{2}}{2}}
$$

and

$$
\|\mathbf{x}+\mathbf{y}\|=\sqrt{\frac{\left(X_{\mathbf{u}}+Y_{\mathbf{u}}\right)^{2}}{2}+{\frac{\left(X_{\mathbf{v}}+Y_{\mathbf{v}}\right)^{2}}{2}}^{2}+\frac{\left(X_{\mathbf{u}}+Y_{\mathbf{u}}+X_{\mathbf{v}}+Y_{\mathbf{v}}\right)^{2}}{2}} .
$$

For vectors

$$
\left(X_{\mathbf{u}}, X_{\mathbf{v}}, X_{\mathbf{u}}+X_{\mathbf{v}}\right), \quad\left(Y_{\mathbf{u}}, Y_{\mathbf{v}}, Y_{\mathbf{u}}+Y_{\mathbf{v}}\right)
$$

and their sum

$$
\left(X_{\mathbf{u}}+Y_{\mathbf{u}}, X_{\mathbf{v}}+Y_{\mathbf{v}}, X_{\mathbf{u}}+X_{\mathbf{v}}+Y_{\mathbf{u}}+Y_{\mathbf{v}}\right),
$$

the triangle inequality in Euclidean space gives

$$
\begin{aligned}
& \sqrt{\left(X_{\mathbf{u}}+Y_{\mathbf{u}}\right)^{2}+\left(X_{\mathbf{v}}+Y_{\mathbf{v}}\right)^{2}+\left(X_{\mathbf{u}}+Y_{\mathbf{u}}+X_{\mathbf{v}}+Y_{\mathbf{v}}\right)^{2}} \\
& \leqslant \sqrt{X_{\mathbf{u}}^{2}+X_{\mathbf{v}}^{2}+\left(X_{\mathbf{u}}+X_{\mathbf{v}}\right)^{2}} \\
& \quad+\sqrt{Y_{\mathbf{u}}^{2}+Y_{\mathbf{v}}^{2}+\left(Y_{\mathbf{u}}+Y_{\mathbf{v}}\right)^{2}} .
\end{aligned}
$$

Dividing this inequality by $\sqrt{2}$, it is easy to see that

$$
\|\mathbf{x}+\mathbf{y}\| \leq\|\mathbf{x}\|+\|\mathbf{y}\|
$$

(xi) A numeric verification by Definition 5 . 


\section{A THREE DIMENSIONAL MODIFICATION OF THE GAUSSIAN NUMBER FIELD}

\section{Possible applications}

An advances generalized of complex numbers $\mathbb{C}$ to $\mathbb{R}^{n}, n \geq 3$, with applications to mathematical physics can be found in [6]. The authors used grouptheoretical methods, more exactly cyclic groups $C_{n}$ to "complexify" $\mathbb{R}^{n}$.

Intended applications of mathematical result of the present paper are limited to three dimensions and modelling of human colour vision. Other direction of our possible applications is mathematical modelling of pipe organ sound (in connection to real spacial acoustics, mensuration of pipes, generalized Pythagorean tuning which depends also on musical timbre), cf. grant VEGA 2/0106/19 (The wooden pipe fund of historical organ positives on Slovakia, 2019-2022).

Acknowledgements 1. The authors are grateful to the referee for careful reading of the paper and valuable suggestions and comments.

\section{REFERENCES}

[1] ALPAY, A.-VAJIAC, A.-VAJIAC, M.B.: Gleason,s problem associated to a real ternary algebra and applications, Adv. Appl. Clifford Algebr. 28 (2018), no. 2, pp. 16. (Published online, paper no. 43)

[2] DROZD, YU.A.-KIRICHENKO, V. V.: Finite Dimensional Algebras. Springer-Verlag, Berlin, 1994.

[3] GREGOR, T.-HALUŠKA, J.: Lexicographical ordering and field operations in the complex plane, Stud. Mat. 41 (2014), no. 2, 123-133.

[4] HALUŠKA, J.: On fields inspired with a polar HSV-RGB theory of colour. In: Modern Real Analysis (J. Hejduk, S. Kowalczyk, R. Pawlak, M. Turowska, ed.), Wydawnictwo Universytetu Łódźkiego, Łódź 2015, pp. 69-88.

[5] HARKIN, A.A.-HARKIN, J.B.: Geometry of general complex numbers, Mathematics magazine, 77 (2004), no. 2, 118-129; https://www.researchgate.net/publication/265769569_Geometry_of_Generalized_ complex_Numbers

[6] LIPATOV L. N.-RAUSCH DE TRAUTENBERG, M.-VOLKOV G. G.: On the ternary complex analysis and its application, J. Math. Phys. 49 (2008), no. 1, 013502. (Published online) https://doi.org/10.1063/1.2827469

[7] NECHAEV, V. I.: Number Systems. Izdat. Prosveshchenie, Moscow, 1975. (In Russian)

[8] RUDIN, W.: Functional Analysis (2nd edition). In: McGraw-Hill Series in Higher Mathematics, McGraw-Hill Book Co., New York 1973; In: Series in Pure and Applied Mathematics, McGraw-Hill, Inc., New York, 1991.

[9] PIERCE, R. S.: Associative Algebras. Springer-Verlag, Berlin, 1982. 


\section{JÁN HALUŠKA, MAtGORZATA JASTRZĘBSKA}

[10] SNYDER, H.H.: An introduction to theories of regular functions on linear associative mathematics, In: Commutative Algebra. Analytic Methods (Fairfax, Va., 1979), pp. 75-94; In: Lecture Notes in Pure and App. Math. Vol. 68, Dekker, New York, 1982.

[11] WARNER, S.: Topological Fields. In: Mathematical Studies Vol. 157. In: Notas Mathematica Vol. 126 (L. Nachbin, ed.), Nord-Holland-Amsterdam -N.Y.-Oxford-Tokyo, 1989.

[12] arxiv 1901.08448

Received October 3, 2019

Mathematical Institute

Slovak Academy of Sciences

Grešákova 6

Košice

SLOVAKIA

E-mail: jhaluska@saske.sk

Institute of Mathematics and Physics

Siedlce University

of Natural Sciences and Humanities

3 Maja 54

08-110 Siedlce

POLAND

E-mail: majastrz2@wp.pl 